\title{
Correction to: Vibrio chemaguriensis sp. nov., from Sundarbans, Bay of Bengal
}

\author{
Anwesha Ghosh ${ }^{1}$. Punyasloke Bhadury ${ }^{1}$
}

Published online: 5 March 2021

(c) Springer Science+Business Media, LLC, part of Springer Nature 2021

\section{Correction to: Current Microbiology (2019) 76:1118-1127 https://doi.org/10.1007/s00284-019-01731-7}

The authors would like to correct the errors in the publication of the original article. The corrected details are given below.

(a) Page 1124: "Description of SBOTS_Iso1 sp. nov" should be changed to "Description of Vibrio chemaguriensis sp. nov."

(b) Page 1124: "che.ma' gu. ri. en' sis. N.L. adj.chemaguriensis" should be changed to "che.ma. gu.ri.en'_sis. N.L. masc.adj. chemaguriensis"

(c) Page 1124: "The type strain, Iso1 (= AGPB $001=$ MTCC $12900^{\mathrm{T}}=\mathrm{JCM} 33348^{\mathrm{T}}$ ), was isolated from Chemaguri creek in Sundarbans, India" to be shifted to the end of the protologue on Page 1125 .

(d) Page 1124: "It forms" to be changed to "The species forms"

(e) Page 1124: "The isolate was susceptible to chloramphenicol, ampicillin and streptomycin but was resistant to amphotericin B and ciprofloxacin" to be changed to "The species is susceptible to chloramphenicol, ampicillin and streptomycin but is resistant to amphotericin B and ciprofloxacin"

(f) Page 1125: "It could utilize indole, starch, dextrose, maltose, fructose, galactose, sucrose, mannose, inulin, sodium gluconate, mannitol, cellubiose and lysine" should be changed to "It can utilize tryptophan, starch,

The original article can be found online at https://doi.org/10.1007/ s00284-019-01731-7.

Punyasloke Bhadury

pbhadury@iiserkol.ac.in

1 Integrative Taxonomy and Microbial Ecology Research Group, Department of Biological Sciences, Indian Institute of Science Education and Research Kolkata, Nadia, Mohanpur, West Bengal 741246, India dextrose, maltose, fructose, galactose, sucrose, mannose, inulin, sodium gluconate, mannitol and cellobiose (Table 1)."

(g) Page 1125: "The FAME profile was dominated by even chained fatty acids (31.7\%) with 16:0 and 18:1 $\omega 7 \mathrm{c}$ which were also the highest forms of saturated and unsaturated fatty acids respectively" should be changed to "The FAME profile is dominated by even chained fatty acids with $16: 0$ and $18: 1 \omega 7 \mathrm{c}$ carbon chains which were also the highest forms of saturated and unsaturated fatty acids respectively (Table 2)".

(h) Page 1125: "by even chained fatty acids (31.7\%)" should be changed to "by even chained fatty acids" with the percentage value removed

(i) Page 1125: "SBOTS_Iso1 has a genome" should be changed to "Vibrio chemaguriensis has a genome"

(j) Page 1125: "sugars and amino acids, biofilm formation and multidrug resistance" to be changed to sugars and amino acids, biofilm formation and drug resistance"

(k) Page 1125: "In silico results showed the organism to use carbon sources including sucrose, D-mannose, trehalose for growth" to be removed

(l) Page 1125: "Amino acids such ornithine, arginine and lysine utilization were shown to be positive" should be removed

(m) Page 1125: "Enzymes including ornithine decarboxylase (EC4.1.1.17), lysine decarboxylase (EC4.1.1.18) and arginine decarboxylase (EC4.1.1.19) are encoded by the genome" should be removed

Publisher's Note Springer Nature remains neutral with regard to jurisdictional claims in published maps and institutional affiliations. 